\title{
Reflux Condensation with Various Non-Condensable Gases
}

\author{
M. Szogradi ${ }^{1,2}$, F. Janasz ${ }^{3}$, Choi Ki-Yong ${ }^{1,2}$ \\ ${ }^{1}$ Thermal-Hidraulics Safety Research Division, Korea Atomic Energy Research Institute \\ P.O. Box 105, Yuseong-gu, Daejeon 34018, Republic of Korea \\ ${ }^{2}$ University of Science and Technology \\ 176 Gajeong-dong, Yuseong-gu, Daejeon, 305-500, Republic of Korea \\ marton.szogradi@psi.ch \\ ${ }^{3}$ Laboratory for Thermal-Hydraulics, Paul Scherrer Institut \\ Villigen 5232, Switzerland
}

\begin{abstract}
Reflux condensation experiments were conducted in the PRECISE facility in order to study the effects of non-condensable (NC) gas type $\left(\mathrm{N}_{2}\right.$ or $\left.\mathrm{He}\right)$ as well as pressure. The facility is a vertical co-current heat exchanger composed of a closed inner test tube and outer coolant channel. The facility is equipped with instrumentation providing data on vertical and horizontal temperature distributions, operating pressure, liquid film behaviour and wall heat fluxes. The conclusions of these experiments can contribute to the condensation model development in CFD codes and nuclear system safety codes.
\end{abstract}

Keywords: Reflux condensation, non-condensable, vertical tube, gas plugging

\section{Introduction}

In the last decade studies focused on alternative heat removal capabilities of nuclear systems. During mid-loop operation (shutting down/starting reactor unit) accident scenarios the reflux condensation can be exploited to maintain a safe steady state in a nuclear primary system.

Vierow et al. [1] performed tests on vertical co-current heat exchanger with $19.3 \mathrm{~mm}$ inner tube diameter under 1-4 bar operating pressure (OP) and 0.014-0.2 air mass fractions. Empirical correlations were derived for the Nusselt number and condensation heat transfer coefficient where the effect of turbulence was taken into account via the Reynolds number. Steam-air mixture temperature distribution was calculated with these correlations and showed a relatively good agreement with measurements. The heat flux estimation from steam-air mixture was validated by this method however further experimental data was required to cover a wider range of boundary conditions.

Lee et al. [2] conducted experiments with a single U-tube with $16.2 \mathrm{~mm}$ inner diameter and $2.8 \mathrm{~m}$ height. The operating pressure was 1 bar and the non-condensable (NC) gas was air with mass fraction of 0.02-0.83. The U-tube was placed in a water tank thus natural convection provided cooling. Since test pressures were atmospheric the results could not describe the condensation phenomena adequately for our purposes. In order to address this issue experimental data was compiled with data from Moon's [3] experiments. Comparing measured and calculated heat transfer coefficients with the Nusselt theory a degradation factor was developed as a function of air mass fraction, Reynolds and Jacob number. Analysing the final results one can see that the root mean square error was $27.4 \%$ between experimental and calculated HTC values which was close to the previous $30 \%$ obtained by Moon. Also the maximum test pressure was 2.5 bar which was still low according to industrial standards where reflux condensation appears at 10 bar during SBLOCA tests (ATLAS facility, KAERI). Furthermore, Moon used forced flow on secondary side with high mass fluxes thus it is difficult to compare it with natural convection cooling applied by Lee.

Dehbi et al. [4] chose a different approach using ANSYS CFD Fluent code to integrate a condensation model for vapor-non-condensable mixture medium. Preliminary assumptions were made to simplify modelling, thus the liquid film thermal resistance was neglected and local thermal equilibrium was assumed at liquid-vapor interface. Suction effects which cannot be modelled directly in CFD owing to the imposed non-slip boundary conditions have been incorporated in an ad-hoc fashion through an enhanced diffusion coefficient. Comparison of simulations to experimental data on open flat 
plate geometries showed relatively good agreement; however, the application of the model to reflux condensation still needs to be validated.

Yi et al. [5] studied the NC effect on steam condensation heat transfer on isothermal vertical aluminum plate under atmospheric pressure. The vapor-air mixture velocity was in a range of $0.1-1 \mathrm{~m} / \mathrm{s}$ with $5-50 \%$ air mass fraction. Results showed that HTC decreased with plate sub-cooling, their values declined greatly with 5-10\% air mass fractions however when the NC mass fraction exceeded $15 \%$ the impact of $\mathrm{NC}$ on condensation rates gradually decreased. The authors explained the tendencies as the heat resistance of the NC layer was relatively stable when air mass fractions were higher. The main part of the effective thermal resistance belonged to the NC layer thus HTC changed slightly when surface subcooling increased.

Fu et al. [6] compared CFD (Fluent) calculation results and test data of condensation in a vertical tube (Din=47.5 mm) with steam-air and steam-helium mixtures under 4 bar OP. Centerline thermocouple standard/maximum deviation was $0.25 / 0.6{ }^{\circ} \mathrm{C}$ respectively, Fluent showed good agreement with experimental data. The diffusion coefficient of steam-helium mixture was $\sim 2.5$ times higher than that of steam-air under same boundary conditions, thus for forced convection flow mass transfer in steam-helium mixture is larger than in steam-air mixture. The difference is significant when steam inlet molar fraction is small, although condensate rates are similar when steam inlet molar fraction is over $90 \%$.

Data presented in this paper can provide required information for further model development. Series of experiments were conducted in the PRECISE facility in order to study the effect of pressure and non-condensable gases on condensation. The first considered pure N2 gas load with various mole fractions on 4 bar operating pressure, the second considered the same N2 mole fractions at 5.65 bar OP and the third considered pure He load with the previously used mole fractions at 4 bar OP. Considering safety regulations He was chosen as a substitute of $\mathrm{H} 2$ to see how light NC gases behave.

\section{PRECISE Facility}

\subsection{General Features}

The facility represents a co-current heat exchanger with a single vertical test tube made of SS2348 alloy. The length and the inner diameter of the test section are $1583 \times 20 \mathrm{~mm}$ respectively. In Fig. 1 one can see the layout of PRECISE facility. Coolant enters the at the bottom and provides cooling for steam vapor of the primary side boiled in the heater tank. Demineralized water and the non-condensable gases are supplied from separate tanks which are connected to the test section.

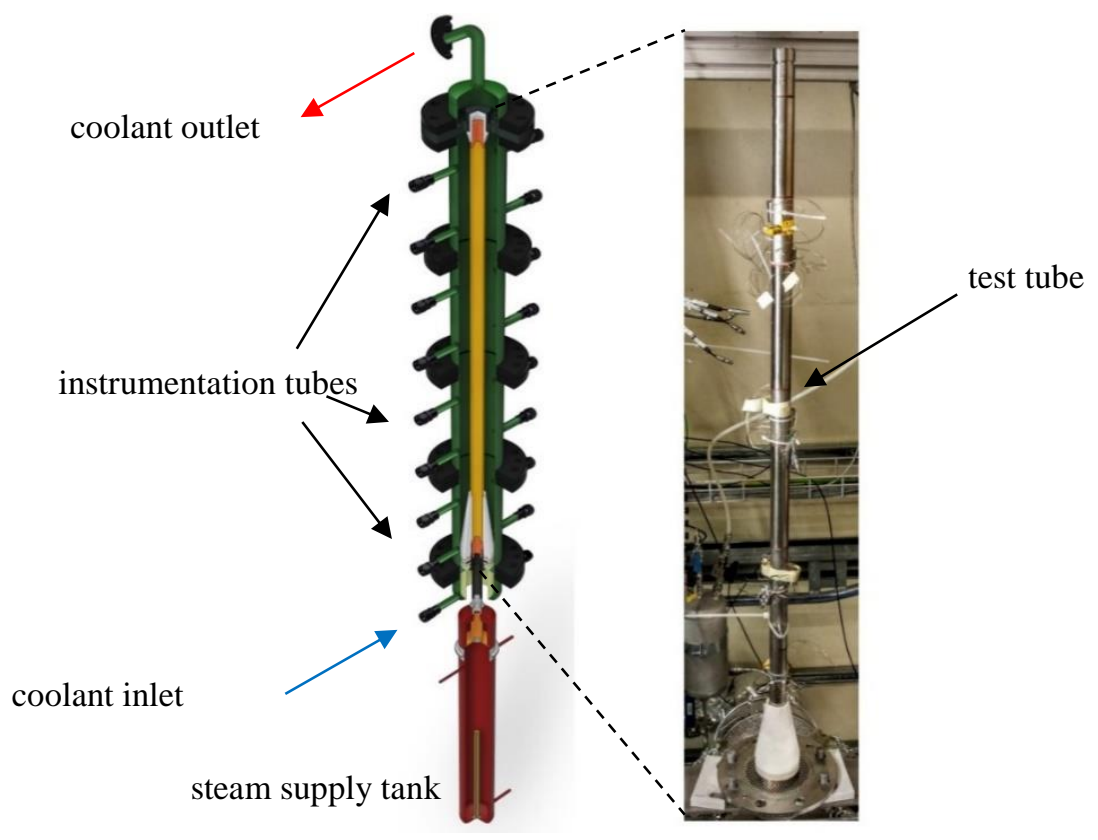

Fig. 1: PRECISE facility layout. 
The maximum heater power is $2 \mathrm{~kW}$ and the maximum OP is 10 bar. The secondary coolant flow used during experiments was set at $2 \mathrm{~m}^{3} / \mathrm{h}$ flow rate to ensure turbulent flow.

\subsection{Instrumentation}

The test section contains $20 \mathrm{pc}$. K-type thermocouples (TC), 11 located in the centre of the test tube (on average one TC every $100 \mathrm{~mm}$ ), 8 record inside and outside test tube wall temperatures and one thermocouple is located on the movable probe. High precision pressure transducers are installed in the test section and non-condensable supply tank for well controlled gas injections.

\subsection{Operational Procedures}

Desired test conditions are set with main parameters as test pressure, NC gas mole fraction, secondary coolant flow rate and desired wall sub-cooling. Based on these values secondary properties are determined such as coolant temperature and pressure. Coolant water can be pressurized up to 10 bars to avoid boiling.

After coolant pre-conditioning step the test section is vacuumed and filled with controlled amount of NC gas mixture and water based on preliminary calculations. Desired test pressure is set and appropriate controls maintain power supply to the main heater as to establish a steady state test conditions.

\section{Results}

\subsection{Nitrogen Tests, 4 Bar OP}

First the pure $\mathrm{N}_{2}$ tests are discussed in this section, the experimental matrix is shown in Table 1 . The first case was a reference test with pure steam medium to represent the most efficient reflux condensation conditions. The third column of the table below shows the $\mathrm{N}_{2}$ mole fraction in the $\mathrm{NC}$ gas mixture. Values between 0 and 1 represents a mixture, 0 means the absence of $\mathrm{N}_{2}, 1$ means pure $\mathrm{N}_{2}$ gas.

Table 1: Pure $\mathrm{N}_{2}$ single injection test matrix at 4 bar OP.

\begin{tabular}{|c|c|c|c|c|}
\hline Test name & $\begin{array}{c}\text { Mole fr NC } \\
{[-]}\end{array}$ & $\begin{array}{c}\mathrm{N}_{2} \text { fr in NC mix } \\
{[\%]}\end{array}$ & $\begin{array}{c}\text { Test absolute } \\
\text { pressure }[\mathrm{b} a r]\end{array}$ & $\begin{array}{c}\text { Wall sub-cooling } \\
{\left[{ }^{\circ} \mathrm{C}\right]}\end{array}$ \\
\hline NC-MFR-ABS-4_1 & 0 & - & 4 & 20 \\
\hline NC-MFR-ABS-4_2 & 0.05 & 1 & 4 & 20 \\
\hline NC-MFR-ABS-4_3 & 0.1 & 1 & 4 & 20 \\
\hline NC-MFR-ABS-4_4 & 0.15 & 1 & 4 & 20 \\
\hline NC-MFR-ABS-4_5 & 0.2 & 1 & 4 & 20 \\
\hline NC-MFR-ABS-4_6 & 0.25 & 1 & 4 & 20 \\
\hline
\end{tabular}

In Fig. 4. the centreline temperature distribution is shown, representing the NC agent effect on vapour temperatures. The highest bulk temperatures can be seen without NC gas; as NC mole fraction increases condensation degrades along the vertical axis. Standard deviations of centreline temperature measurements showed the highest values in the centre of the transition regions, which indicates the most turbulent part of the mixing region. This turbulent zone is the transition between pure water vapour and $\mathrm{NC}$ gas plug. Temperature measurements correspond to steam partial pressure assuming thermal equilibrium thus one can divide the test tube into three sections: vapour volume, mixing region and NC-plugged region (see Fig. 2.) 


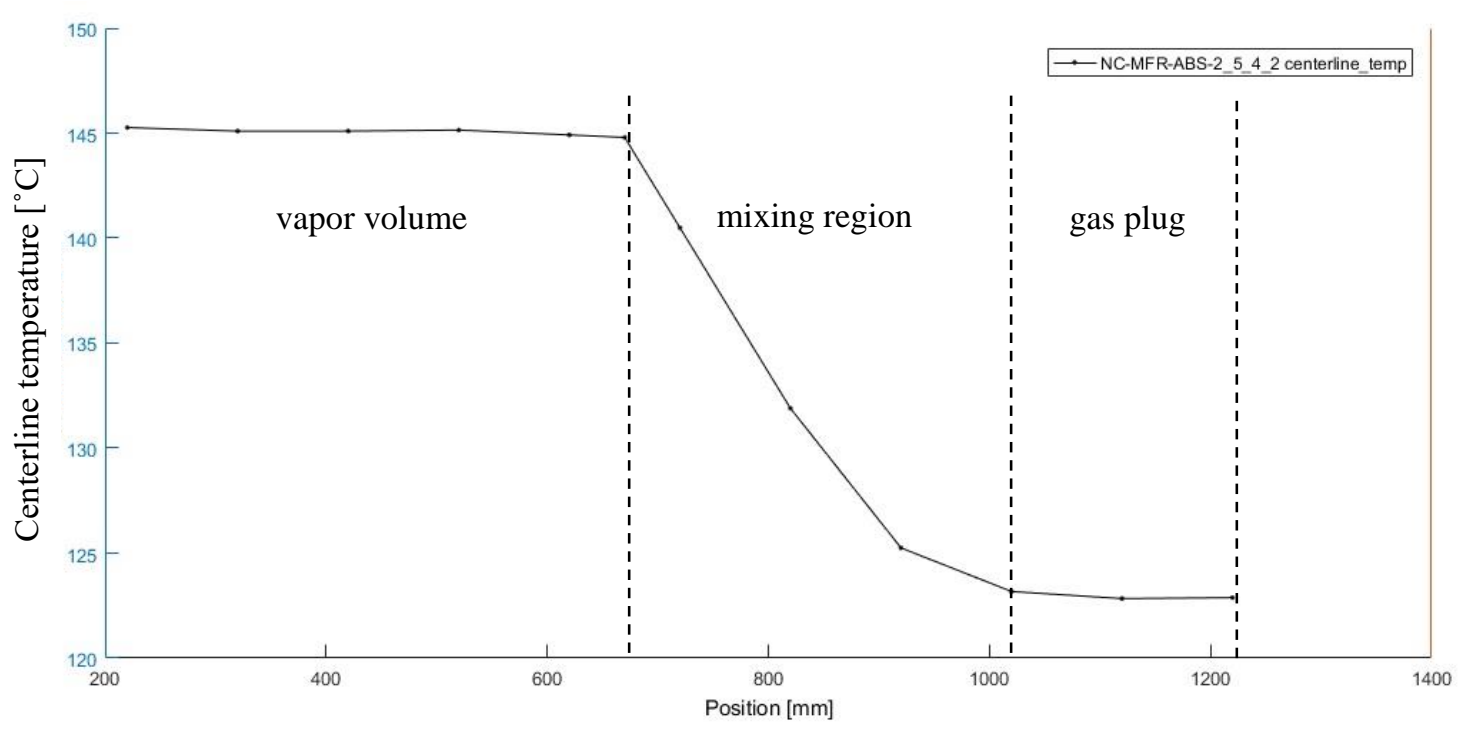

Fig. 2: Test section medium structure.

Considering the TC resolution one can approximate the length of the mixing region with $\sim 400-500 \mathrm{~mm}$. As the gas plug was gradually building up with increasing N2 mole fraction between tests the condensation front descended, finally it could not be observed with even the lowest thermocouple when average $\mathrm{N} 2$ mole fraction was higher than $15 \%$. At this amount of NC gas, calculated steam mass flow dropped by $12.5 \%$ compared to pure steam case. This behavior was also seen with pure He tests. It can be explained by the gas plug building up from the tube top. The NC volume covers a bigger part of the tube inner surface in every subsequent test, instead of creating a uniform mixture with water vapor. As the area available to condensation shrinks the heat transfer rates also decrease resulting in lower condensation rates (see Fig. 3.).

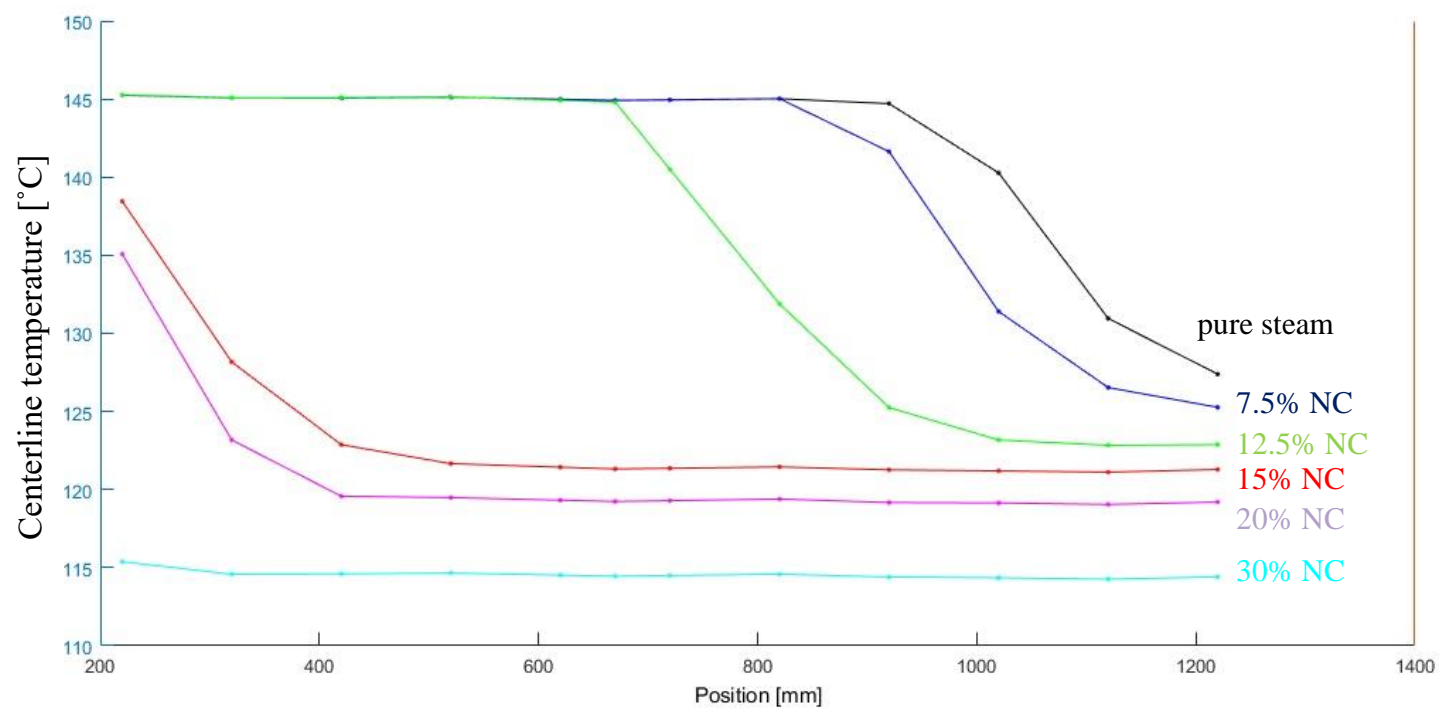

Fig. 3: Centerline temperature distribution of pure $\mathrm{N}_{2}$ experiments.

Considering the horizontal temperature profiles, one can see that the average temperature drop was $\sim 4^{\circ} \mathrm{C}$ between the bulk and wall regions is a reasonable value considering wall sub-cooling of $20^{\circ} \mathrm{C}$. One can see that the temperature starts to decrease approximately at $2.4 \mathrm{~mm}$ from the wall. 


\subsection{Nitrogen Tests, 5.65 Bar OP}

Experiments were conducted with the same $\mathrm{N}_{2}$ mole fractions at a slightly elevated pressure of 5.65 bar (see Table 2.) to verify the gas plug behavior. Since the heater power was insufficient to maintain a $20^{\circ} \mathrm{C} \Delta \mathrm{T}$ at 6 bar OP steady state was maintained at 5.65 bar.

Table 2: Pure $\mathrm{N}_{2}$ single injection test matrix 5.65 bar OP.

\begin{tabular}{|c|c|c|c|c|}
\hline Test name & $\begin{array}{c}\text { Mole fr NC } \\
{[-]}\end{array}$ & $\begin{array}{c}\mathrm{N}_{2} \text { fr in NC mix } \\
{[\%]}\end{array}$ & $\begin{array}{c}\text { Test pressure } \\
{[\mathrm{bar}]}\end{array}$ & $\begin{array}{c}\text { Wall sub-cooling } \\
{\left[{ }^{\circ} \mathrm{C}\right]}\end{array}$ \\
\hline NC-MFR-ABS-N2-6_1 & 0.05 & 1 & 5.65 & 20 \\
\hline NC-MFR-ABS-N2-6_1_5 & 0.075 & 1 & 5.65 & 20 \\
\hline NC-MFR-ABS-N2-6_2 & 0.1 & 1 & 5.65 & 20 \\
\hline NC-MFR-ABS-N2-6_2_5 & 0.125 & 1 & 5.65 & 20 \\
\hline NC-MFR-ABS-N2-6_3 & 0.15 & 1 & 5.65 & 20 \\
\hline NC-MFR-ABS-N2-6_3_5 & 0.175 & 1 & 5.65 & 20 \\
\hline NC-MFR-ABS-N2-6_4 & 0.2 & 1 & 5.65 & 20 \\
\hline
\end{tabular}

Considering centerline temperature profiles there is no significant difference compared to previous experiments. The shape and the length of the mixing zone remained similar compared to 4 bar OP case, thus the mixing did not change as it is shown in Fig. 4. The thermal BL thickness was approximately $2.2 \mathrm{~mm}$, the temperature difference between bulk and inner wall remained at roughly $5^{\circ} \mathrm{C}$.

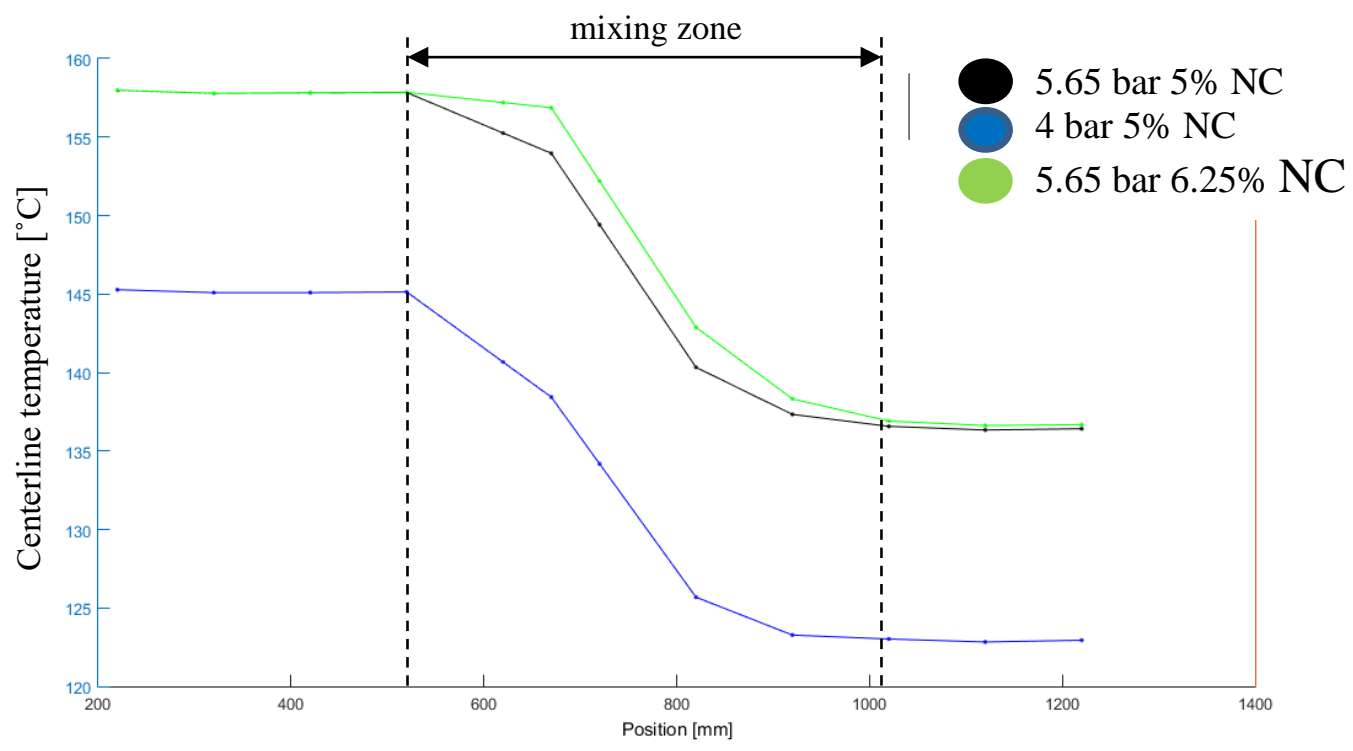

Fig. 4: Temperature distribution of $\mathrm{N}_{2}$ tests on 4 and 5.65 bar OP.

\subsection{Helium Tests, 4 Bar OP}

Pure He tests were conducted at 4 bar OP with the same mole fraction range as the $\mathrm{N}_{2}$ tests. Bulk temperatures showed similar values as before although the mixing zone significantly decreased resulting in a $\sim 300 \mathrm{~mm}$ length as can be seen in Fig. 5. Centreline temperature oscillations also showed lower values which can be explained by lower mixing/turbulence due to smaller molecular weight and more severe stratification. 
Table 3: Pure He single injection test matrix at 4 bar OP.

\begin{tabular}{|c|c|c|c|c|}
\hline Test name & $\begin{array}{c}\text { Mole fr NC } \\
{[-]}\end{array}$ & $\begin{array}{c}\mathrm{N}_{2} \text { fr in NC mix } \\
{[\%]}\end{array}$ & $\begin{array}{c}\text { Test pressure } \\
{[\mathrm{bar}]}\end{array}$ & $\begin{array}{c}\text { Wall sub-cooling } \\
{\left[{ }^{\circ} \mathrm{C}\right]}\end{array}$ \\
\hline NC-MFR-ABS-He-4_1 & 0.05 & 0 & 4 & 20 \\
\hline NC-MFR-ABS-He-4_1_5 & 0.075 & 0 & 4 & 20 \\
\hline NC-MFR-ABS-He-4_2 & 0.1 & 0 & 4 & 20 \\
\hline NC-MFR-ABS-He-4_2_5 & 0.125 & 0 & 4 & 20 \\
\hline NC-MFR-ABS-He-4_3 & 0.15 & 0 & 4 & 20 \\
\hline NC-MFR-ABS-He-4_3_5 & 0.175 & 0 & 4 & 20 \\
\hline NC-MFR-ABS-He-4_4 & 0.2 & 0 & 4 & 20 \\
\hline
\end{tabular}

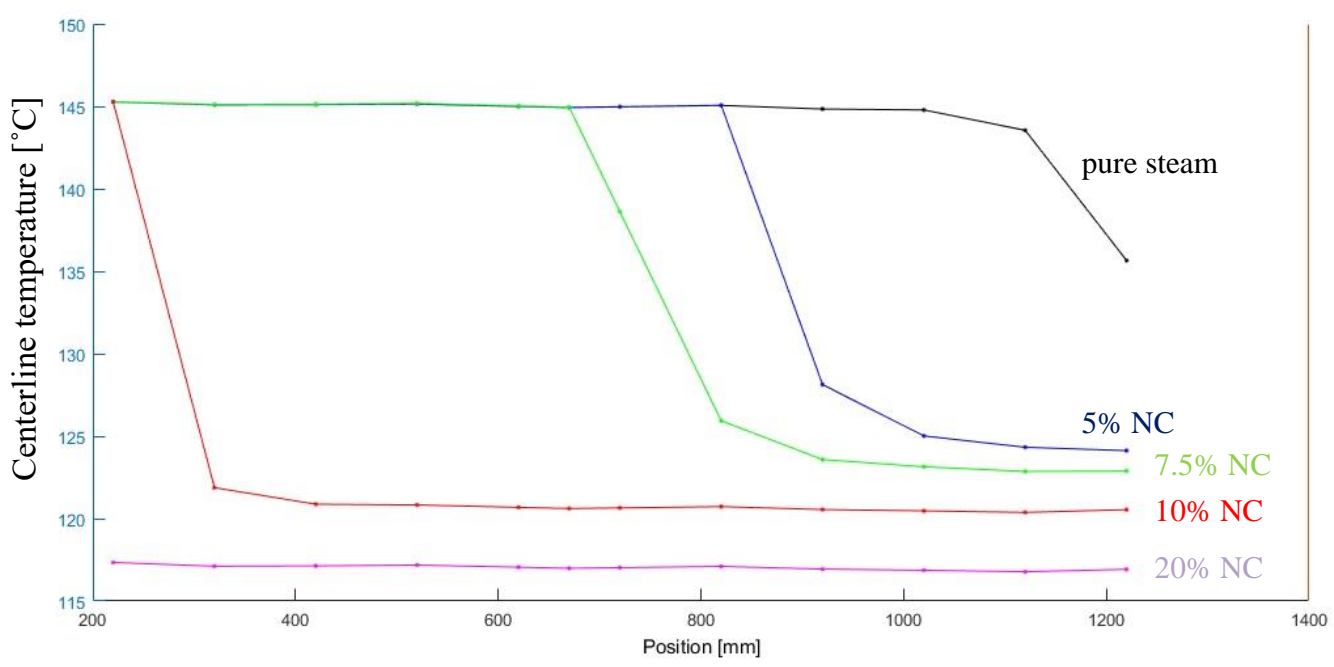

Fig. 5: Temperature distribution during pure He test at 4 bar OP.

Comparing He to $\mathrm{N}_{2}$ steam mass flows we can see a more effective condensation degradation in case of He. This tendency can be observed in condensation fluxes as well as in temperature distributions. At $12.5 \%$ He mole fraction the condensation is stopped while in case of $\mathrm{N}_{2}$ it still continues over 15\% mole fraction. In Fig. 6 one can see the centerline temperature tendencies with the same amount of $\mathrm{N}_{2}$ and $\mathrm{He}$ at 4 bar OP. The secondary $\mathrm{Y}$ axis shows the standard deviations scale; in case of helium the STD values are lower than with nitrogen which indicates the lower level of mixing. Also the length difference of the mixing zones lengths are quite significant - seen as approximately $200 \mathrm{~mm}$. 


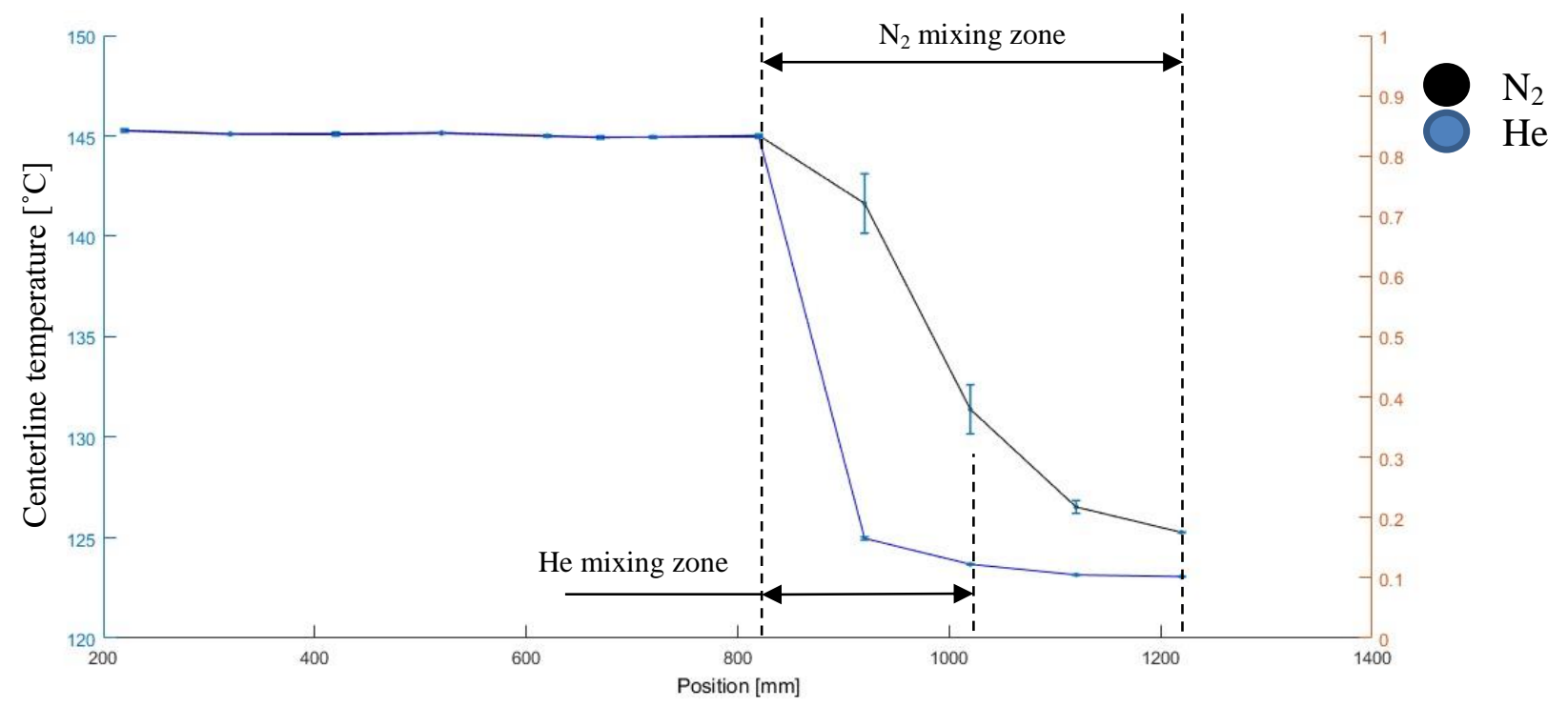

Fig. 6: Centerline temperatures with $7.5 \% \mathrm{NC}$ mole fraction in case of $\mathrm{He}$ and $\mathrm{N}_{2}$ at 4 bar OP.

The mixing behavior differences can be seen between $\mathrm{N}_{2}$ and He representing the centerline thermocouple standard deviations. Comparing the same NC mole fraction (7.5\%) experiments in Fig. 7. nitrogen shows significantly higher STD values in the elevation of 800-1100 mm where we can see center of the mixing zone in Fig. 6.

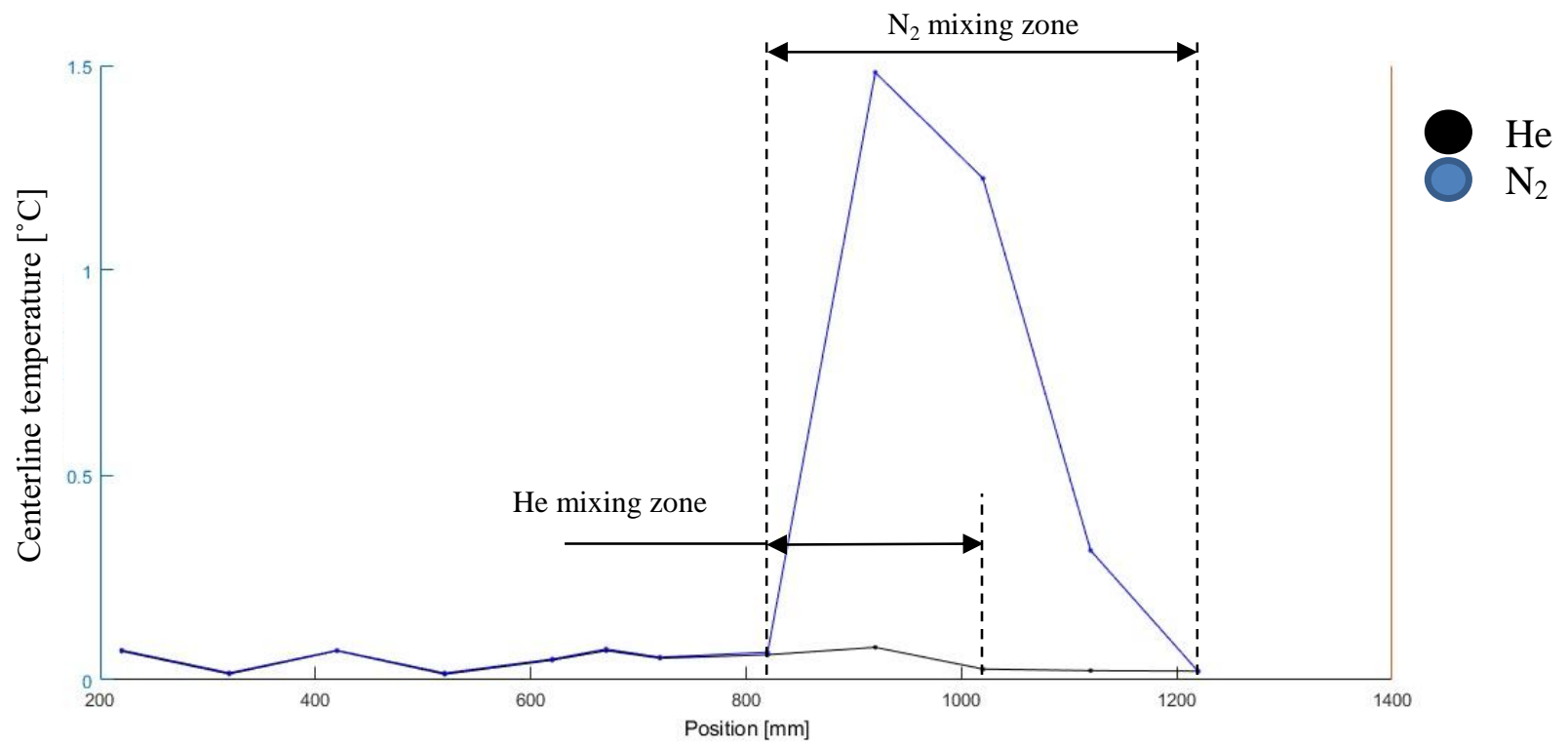

Fig. 7: Centerline temperature STDs with 7.5\% NC mole fraction in case of $\mathrm{He}$ and $\mathrm{N}_{2}$ at 4 bar OP.

One can see in steam mass flow decrease in case of He compared to $\mathrm{N}_{2}$ that $\mathrm{He}$ is a more efficient gas in degradation condensation. This effect can be explained by the diffusion coefficient of He which is four times higher than the air $\left(\mathrm{N}_{2}\right)$ thus it represents a higher insulation in the wall region than $\mathrm{N}_{2}$.

\section{Conclusion}

Successful reflux condensation tests were performed with varying boundary conditions in the presence of NC gases. Pure N2 tests at 4 and 5.65 bar OP showed that the pressure change has no significant effect in the range of 4-5.65 bar on the overall behavior of the gas plug and on the thermal boundary layer although steam flow rates follow the boiling 
temperature tendencies. Small amounts of NC (15\% mole fraction) was enough to significantly degrade condensation in the test tube by covering heat transfer area.

In case of helium tests, one can see that the mixing zone shrunk compared to N2 tests, temperatures showed less deviation with He. Based on recorded steam mass flows, one can conclude that $\mathrm{He}$ is a more effective gas in causing condensation degradation than N2. Based on data and molar weights of these NCs it seems that molecule size has a large role in condensation rates; if one extrapolates the observed tendency even lighter hydrogen gas might be an even more hindering gas to condensation.

\section{Acknowledgements}

The authors would like to express their sincerest gratitude for all the help and guidance from Dr. Abdelouahab Dehbi, Dr. Detlef Suckow, the laboratory staff, and also for the financial support of Paul Scherrer Institut and Korea Atomic Energy Institute which was essential to realize the project.

\section{References}

[1] K. Vierow, T. Nagae, M. Murase, T. Wu, "Evaluation of Reflux Condensation Heat Transfer of Steam-Air Mixtures under Gas-Liquid Countercurrent Flow in a vertical Tube" in Journal of Nuclear Science and Technology, vol. 42, no. 1, pp. 50-57, 2005.

[2] K.-W. Lee, H. C. No, I.-C. Chu, Y. M. Moon, M.-H. Chun, "Local heat transfer during reflux condensation mode un a U-tube with and without noncondensible gases," in International Journal of Heat and Mass Transfer, vol. 49, pp. 1813-1819, 2006.

[3] Y. M. Moon, H. C. No, H. S. Park, Y. S. Bang, "Assessment of RELAP5/MOD3.2 for Reflux Condensation Experiment," International Agreement Report, NUREG/IA-0181, Office of Nuclear Regulatory Research, Washington DC, 2000.

[4] A. Dehbi, F. Janasz, B. Bell, "Prediction of steam condensation in the presence of non-condensable gases using a CFD-based approach," in Nuclear Engineering and Design, no 258, pp 199-210, 2013.

[5] Q. Yi, M. Tian, W. Yan, X. Qu, X. Chen,"Visualization study of the influence of non-condensable gas on steam condensation heat transfer," in Applied Thermal Engineering, vol. 106, pp 13-21, 2016.

[6] W. Fu, X. Li, X. Wu, M. L. Corradini, "Numerical investigation of Convective condensation with the presence of non-condensable gases in a vertical tube," in Nuclear Engineering and Design, vol. 297, pp 197-207, 2016. 